\title{
Simulação numérica para análise de deflexões e tensões em placas finas de aço e de materiais compósitos submetidas a esforços de flexão
}

\author{
Numerical simulation for the analysis of deflections and stresses in thin steel plates and \\ composite materials submitted to bending efforts \\ J. V. de A. Carvalho; D. Helbig* \\ Escola de Engenharia (EE), Universidade Federal do Rio Grande, 96201-900, Rio Grande-RS, Brasil \\ *daniel.helbig@gmail.com
}

\begin{abstract}
As evoluções no desenvolvimento de materiais compósitos possibilitam cada vez mais o seu uso. Algumas das ferramentas que contribuíram para essa evolução foram o Método dos Elementos Finitos e as ferramentas de simulação numérica, pois possibilitaram a avaliação do comportamento e da resistência desses materiais ainda na fase de projeto. Esses métodos permitem ao pesquisador conhecer de maneira muito próxima o comportamento dos elementos e das estruturas, porém, como são métodos aproximados, não se pode esperar que os resultados fossem exatamente iguais aos reais. Este trabalho procura analisar o comportamento mecânico de placas finas de materiais compósitos laminados reforçados por fibras submetidas a esforços de flexão, comparando-as com o de uma placa de aço. Para a modelagem computacional do problema utilizouse o software Abaqus 2017 Student Version ${ }^{\circledR}$ cuja base está fundamentada no Método dos Elementos Finitos. Na sequência, busca-se comparar os resultados obtidos neste trabalho com os encontrados na literatura. A análise dos dados, através de duas diferentes ferramentas computacionais disponíveis no mercado, tem como objetivo verificar a coerência e a equivalência entre os resultados. Pôde-se verificar que os dados obtidos neste trabalho e na literatura no software Ansys ${ }^{\circledR}$ apresentaram equivalência entre si e deles para com a solução analítica desenvolvida para o problema.

Palavras-chave: Simulação Numérica, Modelagem Computacional, Materiais Compósitos.
\end{abstract}

The evolution in the development of composite materials increases their use. Some of the tools that contributed to this evolution were the Finite Element Method and numerical simulation tools, as they enabled the evaluation of the behavior and resistance of these materials still in the design phase. These methods allow the researcher to know very closely the behavior of the elements and structures, but as they are approximate methods, the results can not be expected to be exactly the same as the real ones. This work aims to analyze the mechanical behavior of thin sheets of composite materials reinforced by fibers subjected to bending efforts, comparing them with that of a steel plate. For the computational modeling of the problem we used the software Abaqus 2017 Student Version ${ }^{\circledR}$ based on the Finite Element Method. The results obtained in this work are compared with those found by literature. The data analysis, through two different computational tools available in the market, aims to verify the consistency and the equivalence between the results. It could be verified that the data obtained in this work and by literature in Ansys ${ }^{\circledR}$ software presented equivalence among them and with them to the analytical solution developed for the problem.

Keywords: Numerical Simulation, Computational Modeling, Composite Materials.

\section{INTRODUÇÃO}

Os avanços nas áreas de ciências dos materiais proporcionam o uso mais consciente e efetivo dos materiais. Nesse contexto encontram-se os materiais compósitos, dos quais se consegue extrair propriedades úteis a diversas áreas da Engenharia. Esses são formados por laminações de diferentes materiais, tais como polímeros, materiais fibrosos e ligas metálicas. Os Fiber Reinforced Polymer Composites (FRPs) ganham espaço e sua utilização em placas finas passa a ser uma possibilidade interessante.

Através da simulação numérica consegue-se aferir quais os pontos críticos de modelos desenvolvidos com esses materiais. Esta estuda as tensões e deformações ao longo do modelo e avalia qual a resistência em flexão e rigidez [1]. Um dos métodos de simulação numérica é o Método dos Elementos Finitos (MEF), utilizado em diversos softwares, dentre os quais: o Abaqus ${ }^{\circledR}$ 
e o Ansys ${ }^{\circledR}$. O erro é inerente a todos os métodos numéricos. Ainda assim, é útil a avaliação dos resultados em duas ferramentas diferentes a fim de que se compreenda melhor os dados obtidos e se possa ter uma avaliação se esses apresentam equivalência.

Serão realizadas simulações numéricas, com o auxílio do Abaqus®, com o objetivo de analisar e comparar o comportamento mecânico de placas finas de aço e de materiais compósitos laminados reforçados por fibras submetidos a esforços de flexão. Os resultados servirão para a comparação com os obtidos por Helbig et al. (2013) [2], que faz uso do Ansys ${ }^{\circledR}$, os quais espera-se que estes possam ser equivalentes.

\section{MATERIAIS COMPÓSITOS}

Materiais compósitos são aqueles em que dois ou mais materiais são combinados com o objetivo de constituir um terceiro material útil. Esses materiais são comumente classificados como: materiais compósitos fibrosos, materiais compósitos laminados, materiais compósitos particulados e combinações dos três tipos [3].

Esses materiais podem ser constituídos através de ligações mecânicas ou químicas e tipicamente são encontrados na forma de inclusões suspensas em uma matriz, elemento responsável pela transmissão dos esforços para as fibras. O reforço por sua vez tem impacto direto nas características mecânicas do material e na sua fabricação. Os materiais constituintes mantêm suas características e, normalmente, é possível identificá-los fisicamente, de forma que existe uma interface entre eles [4].

Existem, por exemplo, matrizes metálicas, cerâmicas, de carbono e cimentícias, embora as mais utilizadas sejam as poliméricas. Os materiais compósitos de matriz polimérica podem ser divididos em duas categorias principais: as termoplásticas e as termo-endurecíveis. Os reforços, por sua vez, dividem-se em três categorias. Existem materiais reforçados por fibras (como os utilizados para o desenvolvimento deste trabalho), compósitos particulados e compósitos estruturais [1].

Segundo Jones (1999) [3], os principais benefícios na utilização desses materiais estão associados à resistência e rigidez. Normalmente, essas características são expressas em termos relativos, dividindo-as pela densidade. Os valores para as propriedades dos materiais constituintes variam de acordo com o ângulo de colocação das fibras entre $0^{\circ}$ (no sentido das fibras, cujo valor é máximo) e $90^{\circ}$ (perpendicular às fibras, cujo valor é mínimo).

\section{MODELO COMPUTACIONAL}

Com o objetivo de simular o comportamento mecânico de placas finas para diferentes materiais e composições, desenvolveu-se no Abaqus ${ }^{\circledR}$, cuja base é o MEF, o modelo computacional. O MEF consiste na divisão do domínio de integração, chamada rede de elementos finitos, e os pontos de interseção entre as linhas da malha são denominados nós [5]. O comportamento de cada elemento é determinado de forma que a malha se comporte da maneira mais semelhante possível ao contínuo original [2].

Para a modelagem computacional, foi utilizado o elemento S8R: elemento quadrilateral, com 8 nós, análise de deformações e tensões convencional e integração reduzida. Para o S8R podem ser determinadas camadas de materiais compósitos, suas propriedades mecânicas, assim como a espessura e a angulação das diferentes camadas interpostas. Este é composto de 8 nós e 6 graus de liberdade, deslocamento nos eixos $\mathrm{x}$, y e z e rotação nos eixos x, y e z [6].

A definição da malha para a análise do problema é fator crucial para a obtenção de bons resultados. Neste trabalho, a malha será formada por 800 nós, para efeito de comparação com os resultados obtidos por Helbig et al. (2013) [2]. Ressalte-se que a quantidade de nós acima especificada possibilita a utilização da versão estudantil do software Abaqus® (Abaqus 2017 Student Version $\left.{ }^{\circledR}\right)$, limitada a 1000 nós.

O modelo computacional utilizado analisará as tensões e as deflexões em placas finas engastadas nas quatro bordas e submetidas a um carregamento uniforme distribuído. Este apresenta as mesmas características utilizadas em Helbig et al. (2013) [2], o que possibilita a comparação dos resultados. 


\section{VERIFICAÇÃO DO MODELO}

Para a verificação do modelo computacional foi utilizado uma placa de aço, isotrópica, quadrada de $0,50 \mathrm{~m}$ de lado, com espessura de 5,00 x $10^{-3} \mathrm{~m}$ e engastada nas quatro bordas. Para o aço, foram consideradas as propriedades: módulos de elasticidade $\mathrm{E}=210,00 \mathrm{GPa}$ e $\mathrm{G}=80,80 \mathrm{GPa}$ e coeficiente de Poisson $\mu=0,30$. Foi aplicado sobre a placa um carregamento uniforme distribuído de 100,00 Pa. Adotou-se para a simulação numérica o elemento S8R do Abaqus ${ }^{\circledR}$, utilizado para o cálculo de placas e cascas, considerando-se uma placa formada por quatro camadas de igual espessura, para efeito de comparação com o resultado analítico de Timoshenko et al. (1959) [7] para o cálculo da máxima deflexão. A solução analítica apresenta uma deflexão máxima de 3,276 x $10^{-3} \mathrm{~m}$ e, a numérica, de $3,287 \times 10^{-3} \mathrm{~m}$, ou seja, uma diferença de $0,33 \%$, o que verifica o modelo computacional proposto.

\section{RESULTADOS E DISCUSSÃO}

Utilizou-se a simulação numérica, com o auxílio do Abaqus®, para comparar o comportamento mecânico de placas finas de aço e de materiais compósitos laminados reforçados por fibras com os de Helbig et al. (2013) [2]. Considerou-se, como domínio computacional, uma placa de dimensões $(0,20 \times 0,10 \times 0,01) \mathrm{m}$ respectivamente comprimento, largura e espessura, como podem ser visto na Figura 1. Esse foi discretizado por uma malha quadrada de tamanho $5,00 \times 10^{-3} \mathrm{~m}$, com 800 elementos do tipo shell S8R. A placa está engastada nas quatro bordas e submetida a um carregamento uniforme distribuído de 0,20 MPa.

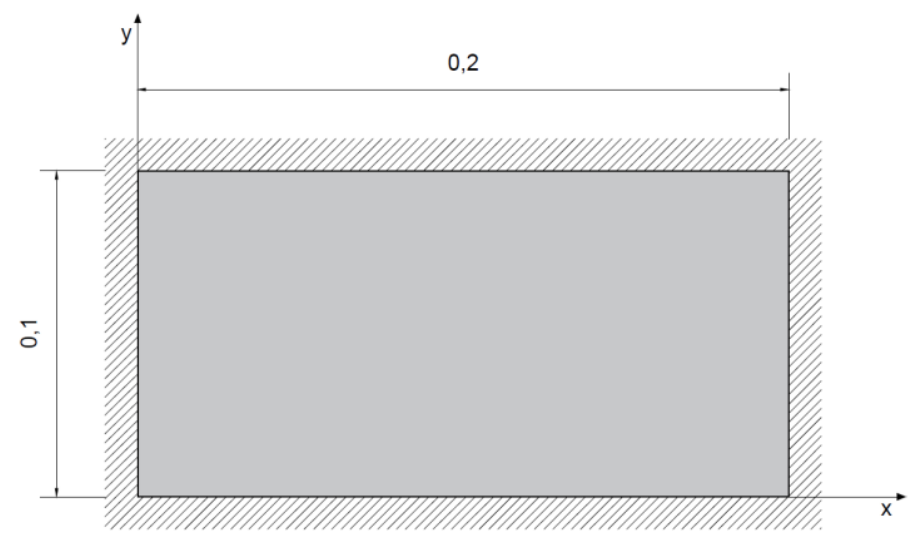

Figura 1: Domínio computacional.

As propriedades mecânicas para o aço e para os materiais compósitos, retirados de Helbig et al. (2013), são apresentadas na Tabela 1 e os esquemas de laminação na Tabela 2.

Tabela 1: Propriedades dos materiais para o aço e os materiais compósitos. Retirado de Helbig et al. (2013) [2].

\begin{tabular}{cccccc}
\hline & \multicolumn{5}{c}{ Materiais } \\
\cline { 2 - 6 } Propriedades & $\begin{array}{c}\text { Aço } \\
(\mathbf{A})\end{array}$ & $\begin{array}{c}\text { Vidro E/ Epóxi } \\
(\mathbf{B})\end{array}$ & $\begin{array}{c}\text { Boro/ Epóxi } \\
(\mathbf{C})\end{array}$ & $\begin{array}{c}\text { Tecido de } \\
\text { Vidro/Epóxi (D) }\end{array}$ & $\begin{array}{c}\text { Boro/ Alumínio } \\
(\mathbf{E})\end{array}$ \\
\hline $\boldsymbol{E}_{\mathbf{x}}(\mathbf{G P a})$ & 210,00 & 39,00 & 201,00 & 29,70 & 235,00 \\
$\boldsymbol{E}_{\mathbf{y}}(\mathbf{G P a})$ & 210,00 & 8,60 & 21,70 & 29,70 & 137,00 \\
$\boldsymbol{E}_{\mathbf{z}}(\mathbf{G P a})$ & 210,00 & 8,60 & 21,70 & 29,70 & 137,00 \\
$\boldsymbol{G}_{\mathbf{x y}}(\mathbf{G P a})$ & 80,80 & 3,80 & 5,40 & 5,30 & 47,00 \\
$\boldsymbol{G}_{\mathbf{y z}}(\mathbf{G P a})$ & 80,80 & 3,80 & 5,40 & 5,30 & 47,00 \\
$\boldsymbol{G}_{\mathbf{z x}}(\mathbf{G P a})$ & 80,80 & 3,80 & 5,40 & 5,30 & 47,00 \\
$\boldsymbol{\mu x y}$ & 0,30 & 0,28 & 0,17 & 0,17 & 0,30 \\
$\boldsymbol{\mu y z}$ & 0,30 & 0,28 & 0,17 & 0,17 & 0,30 \\
$\boldsymbol{\mu z} \boldsymbol{x}$ & 0,30 & 0,28 & 0,17 & 0,17 & 0,30 \\
\hline
\end{tabular}


Tabela 2: Casos e laminações. Retirado de Helbig et al. (2013) [2].

\begin{tabular}{ccc}
\hline Caso & Laminação & Materiais \\
\hline $\mathbf{1}$ & - & $(\mathrm{A})$ \\
$\mathbf{2}$ & {$[0 / 90 / 90 / 0]$} & $(\mathrm{B}),(\mathrm{C}),(\mathrm{D}) \mathrm{e}(\mathrm{E})$ \\
$\mathbf{3}$ & {$[90 / 0 / 0 / 90]$} & $(\mathrm{B}),(\mathrm{C}),(\mathrm{D}) \mathrm{e}(\mathrm{E})$ \\
$\mathbf{4}$ & {$[0 / 90 / 0]$} & $(\mathrm{B}),(\mathrm{C}),(\mathrm{D}) \mathrm{e}(\mathrm{E})$ \\
$\mathbf{5}$ & {$[90 / 0 / 90]$} & $(\mathrm{B}),(\mathrm{C}),(\mathrm{D}) \mathrm{e}(\mathrm{E})$ \\
$\mathbf{6}$ & {$[45 /-45 /-45 / 45]$} & $(\mathrm{B}),(\mathrm{C}),(\mathrm{D}) \mathrm{e}(\mathrm{E})$ \\
$\mathbf{7}$ & {$[45 /-45 / 45]$} & $(\mathrm{B}),(\mathrm{C}),(\mathrm{D}) \mathrm{e}(\mathrm{E})$ \\
\hline
\end{tabular}

Foram adotados, para os casos 2, 3 e 6, esquemas de laminação compostos por quatro camadas de $2,50 \times 10^{-3} \mathrm{~m}$, e para os casos 4,5 e 7 , laminação antissimétrica com três camadas de $3,33 \times 10^{-3} \mathrm{~m}$ (Figura 2). Cabe salientar que este trabalho não tem como objetivo verificar a resistência do material compósito ou de suas camadas, logo não há considerações quanto à ruptura das fibras ou da matriz.

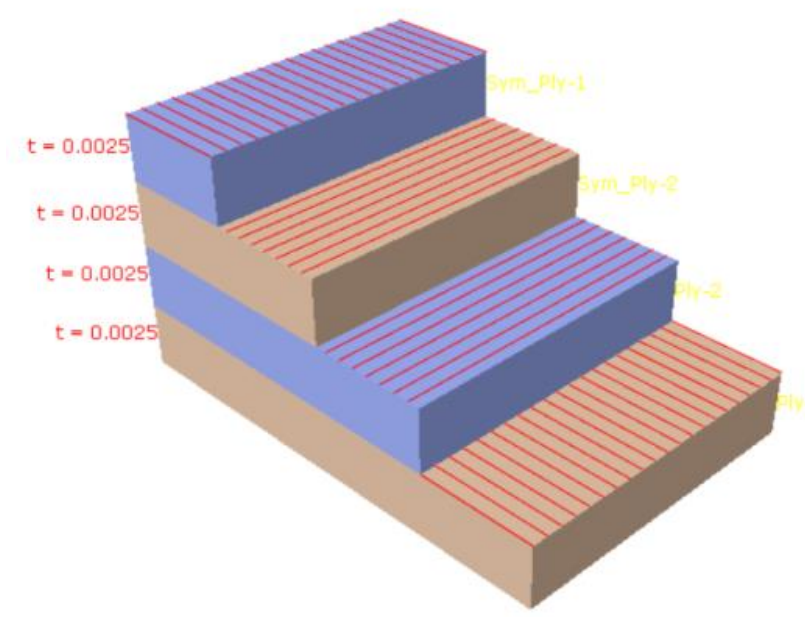

Figura 2: Laminação do caso 2.

A Figura 3a mostra os valores máximos para as deflexões neste trabalho e a Figura $3 \mathrm{~b}$ mostra os de Helbig et al. (2013) [2].
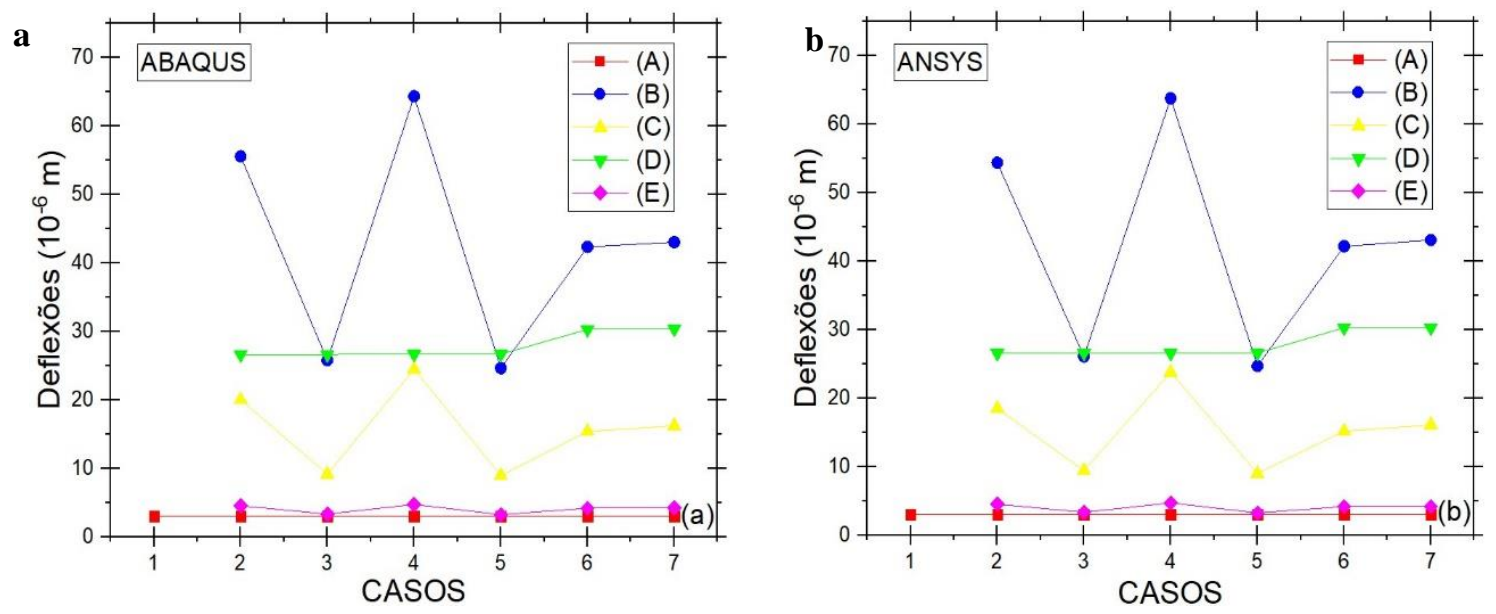

Figura 3: Valores de deflexões máximas - Abaqus ${ }^{(a)}$ e Ansys ${ }^{\circledR}(b)$. 
Observa-se nas Figuras $3 \mathrm{a}$ e $3 \mathrm{~b}$ que os resultados obtidos apresentam comportamento gráfico semelhante aos de Helbig et al. (2013) [2]. Quanto aos resultados numéricos, as maiores diferenças referem-se ao material compósito Boro/Epóxi (C), sendo de 7,68\% e $-2,93 \%$, respectivamente casos 2 e 3.

As Figuras $3^{\mathrm{a}}$ e $3 \mathrm{~b}$ mostram que as menores deflexões, porém maiores que as do aço, referem-se ao material compósito (E) nos casos 3 e 5 . As deflexões máximas foram obtidas para o material compósito (B) e caso 4, no Abaqus ${ }^{\circledR}$ e no Ansys ${ }^{\circledR}$. Quando comparadas com as da placa de aço, são respectivamente $2039,96 \%$ e 2019,60\% superiores. Por outro lado, as mínimas são superiores $8,21 \%$ e $7,97 \%$, respectivamente.

Quando se considera o número de camadas, as máximas referem-se ao caso 4, para os materiais compósitos (B), (C) e (E) com três camadas, e as mínimas ao caso 5 com quatro camadas.

Os valores máximos para as tensões de von Mises são apresentados na Figura 4a e Figura 4b os obtidos por Helbig et al. (2013) [2]. Essa mostra que os resultados encontrados são coerentes com os de Helbig et al. (2013) [2]. As maiores diferenças são de 5,54\% e -2,20\%, respectivamente para o composto Boro/Epóxi (B), caso 2, e Tecido de Vidro/Epóxi (D), caso 6.
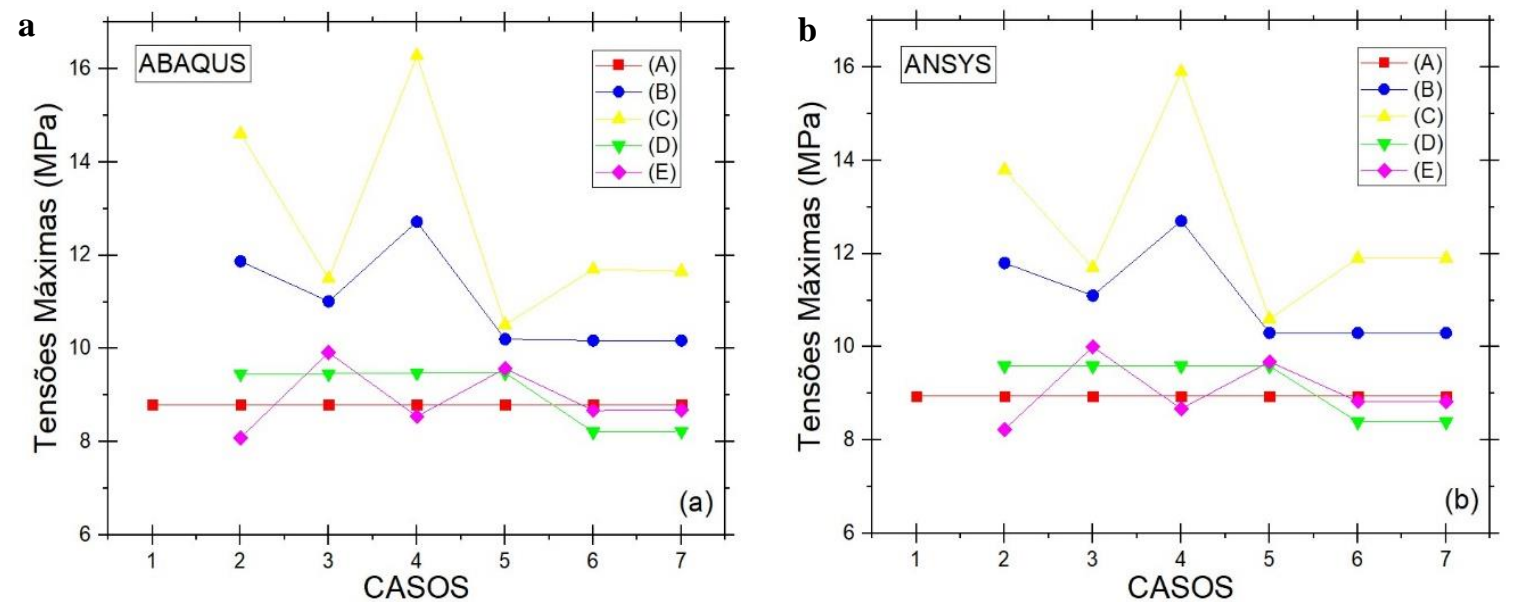

Figura 4: Gráficos de tensões máximas - Abaqus ${ }^{\circledR}(a)$ e Ansys ${ }^{\circledR}(b)$.

Observou-se que apenas os materiais compósitos (E), nos casos 2, 4, 6 e 7, e (D), nos casos 6 e 7 , apresentaram tensões menores que as da placa de aço. Dentre os outros, e para todos os casos considerados, obteve-se valores maiores do que os da placa de aço. Os valores máximos encontrados, em ambos os softwares, foram para o material compósito (C) e caso 4, superiores $85,13 \%$, no Abaqus ${ }^{\circledR}$, e 77,85\%, no Ansys ${ }^{\circledR}$, aos valores para o aço. Por outro lado, as tensões mínimas foram para o material compósito (E) e caso 2, respectivamente 8,06\% e 7,94\% inferiores às da placa de aço. A Figura 5 mostra a distribuição de tensões de von Mises para uma placa de material compósito (C), considerando os casos 4 e 7.
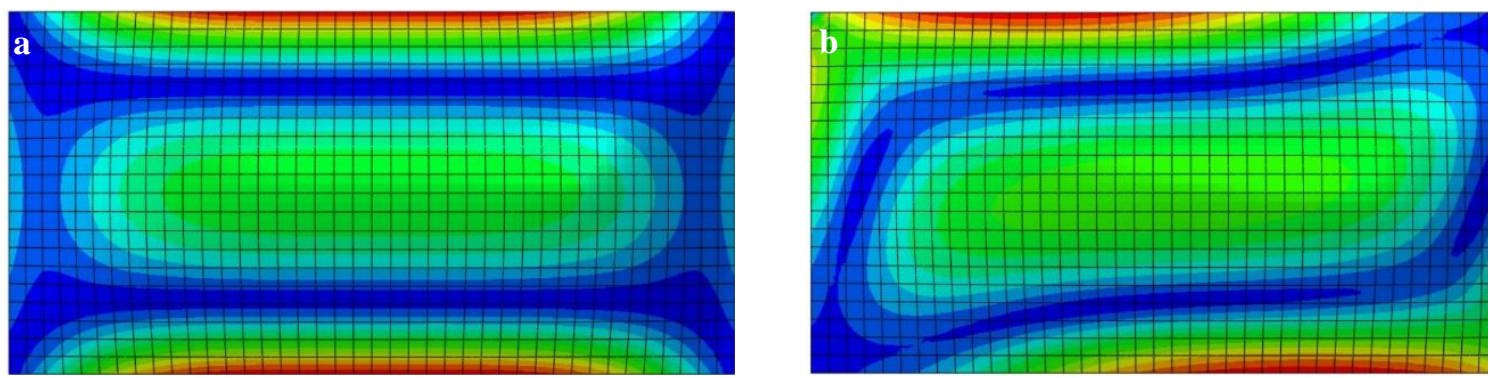

Figura 5: Distribuição de tensões no material compósito $(C)$, caso 4 (a) e caso 7 (b).

Para todos os casos considerados, os maiores valores de tensões correspondem ao material compósito $(C)$. Em relação à orientação das fibras, para os materiais compósitos $(B),(D)$ e (E), foram encontrados valores inferiores quando colocadas à $45^{\circ}$. Quanto à laminação obteve-se para 
os materiais compósitos (B) e (C) valores superiores aos demais nos casos com três camadas e orientação perpendicular das fibras.

\section{CONCLUSÃO}

A crescente utilização de materiais compósitos reforçados por fibras em projetos estruturais os torna tema de grande relevância nas diversas áreas da engenharia.

Foi possível comprovar que placas de material compósito laminado reforçado por fibras apresentam deflexões maiores que as de uma placa de aço. Demonstrou-se que existem situações, casos 2 e 4 para o material compósito (E) e casos 6 e 7 para o material compósito (D), onde as tensões são inferiores às encontradas para uma placa de aço. Logo, considerando que as placas com materiais compósitos laminados reforçados por fibras apresentam menor peso e maior resistência à corrosão, dentre outras vantagens, pode-se afirmar que estes são excelente opção para uso na construção de estruturas navais e offshore, aeroespaciais e na construção civil.

Os resultados obtidos para deflexões e tensões são próximos aos encontrados em Helbig et al. (2013) [2]. Constatou-se também que o comportamento mecânico de placas compósitas laminadas reforçadas por fibras pode sofrer alterações significativas em função da disposição das lâminas e do número de camadas, o que justifica a continuação de pesquisas nesta área.

\section{AGRADECIMENTOS}

Agradecimentos à FURG e à Prof ${ }^{a}$ Raquel Nicolette pela colaboração.

\section{REFERÊNCIAS BIBLIOGRÁFICAS}

1. Teixeira ARP. Design of a composite chassis car [disseratação de mestrado]. Porto (PO): Universidade do Porto; 2014.

2. Helbig D, Silva CCC, Real MDV, Vaz J, Rocha LAO, Santos ED, Isoldi LA. Análise Numérica do Comportamento Mecânico sob Flexão de Placas Finas de Material Compósito Laminado Reforçado por Fibras. Vetor 2013;23(2):5-15.

3. Jones RM. Mechanics of composite Materials. 2nd ed. Blacksburg (United States of America): Taylor \& Francis; 1999. 554 p. Jenkins PF. Making sense of the chest x-ray: a hands-on guide. New York: Oxford University Press; 2005. 194 p.

4. Bower MV. Composite Materials [dissertation]. Huntsville (AL): The University of Alabama in Huntsville; 2000. 200 p.

5. Isoldi L. A. Análise estática e dinâmica de estruturas delgadas de materiais compostos laminados incluindo materiais piezelétricos [tese de doutorado]. Porto Alegre (RS): Universidade Federal do Rio Grande do Sul; 2008. 223 p.

6. Dassault Systemes. Abaqus 2016 online documentation [Computer software manual]. Retirado em Julho 09, 2018 de: http://abaqus.software.polimi.it/v2016/books/usb/default.htm.

7. Timoshenko SP, Woinowsky-Krieger S. Theory of plates and shells. New York (United States of America): McGraw-Hill Book Company; 1959. $591 \mathrm{p}$ 